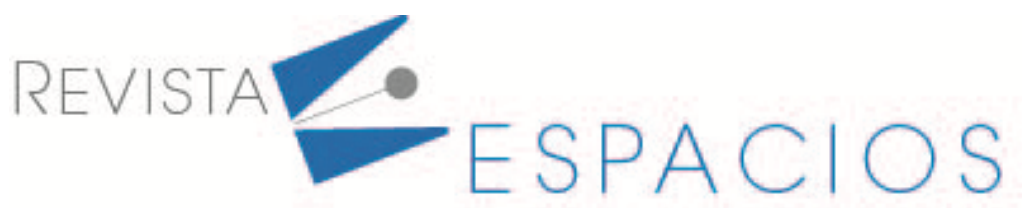

Vol. 42 (08) $2021 \cdot$ Art. 1

Recibido/Received: 10/10/2020 • Aprobado/Approved: 16/01/2021 • Publicado/Published: 30/04/2021

\title{
Estado del arte de los oficios del Paisaje Cultural Cafetero Colombiano (Departamento de Risaralda)
}

\section{State of the art of Colombian Coffee Cultural Landscape crafts (Risaralda Department)}

GÓMEZ, Yaffa N. ${ }^{1}$

LOPEZ, Javier A. ${ }^{2}$

\begin{abstract}
Resumen
La investigación documenta y presenta el estado del arte de los oficios presentes en el Paisaje Cultural Cafetero Colombiano PCCC. En este sentido, el artículo presenta las diversas fuentes donde se realizó la búsqueda de bibliografía, tales como, documentos del Ministerio de Cultura, Biblioteca Banco de la República, Biblioteca Digital de Artesanías de Colombia, así como la página Web del Sistema Nacional de Información Cultural, encontrando documentos estadísticos, trabajos de grado referidos a oficios y caracterización de la población artesanal del PCCC.

Palabras clave: oficios, paisaje cultural cafetero colombiano, cultura material e inmaterial, artesanía.
\end{abstract}

\begin{abstract}
The research documents and presents the state of the art of the trades present in the Colombian Coffee Cultural Landscape PCCC. In this sense, the article presents the various sources where the bibliography search was carried out, such as documents from the Ministry of Culture, Banco de la República Library, Colombia's Digital Handicraft Library, as well as the website of the National Information System Cultural, finding statistical documents, graduate work related to trades and characterization of the artisan population of the PCCC.
\end{abstract}

key words: crafts, colombian coffee cultural landscape, material and intangible culture, handicraft.

\section{Introducción}

La investigación en curso se orienta a dar respuesta a la pregunta de ¿Cuáles son los oficios presentes en el Paisaje Cultural Cafetero Colombiano, PCCC?

Bajo la comprensión de que:

"El oficio se constituye en el contexto dentro del que la creatividad explora todas las posibilidades para la diversificación funcional y/o estética de nuevos productos a partir del sistema materializado en cada taller. El oficio artesanal es arte aplicado, es algo a la vez productivo y creativo. El oficio homogeniza en cierto modo a los factores y rutinas presentes en una serie de talleres, emparentándolos y caracterizándolos desde 
el punto de vista de los factores específicos de producción, de la infraestructura y de las relaciones sociales y técnicas. En el oficio está presente la convergencia de diversos instrumentos, rutinas, acciones, capacidades y resultados". (Bejarano, 2014, p. 37)

El nuevo conocimiento se concentra en la compilación de la información de tres de los departamentos del PCCC en un solo documento, lo cual permitirá mostrar la diversidad o similitud de oficios y productos derivados, así como el reconocimiento de las historias de vida y las técnicas de los artesanos o maestros. Comprendiendo que:

"La técnica es una manera particular de hacer las cosas, de producir un determinado resultado. La técnica puede estar más identificada con destrezas particulares del artesano, con materiales específicos, con rutinas o procesos que se siguen, con el uso de ciertos instrumentos, entre otros aspectos. Sin duda, las técnicas empleadas para la producción de objetos artesanales son tan variadas como los mismos objetos y pueden ir desde procedimientos delicados y minuciosos hasta acciones rudas. Lo característico es que demanden la presencia de factores específicos y que no sean fácilmente replicables". (Bejarano, 2014, p.40)

A nivel local y nacional se cuenta con los documentos que al interior de los Laboratorios de Artesanías de Colombia se han elaborado acerca de los artesanos, técnicas y productos que se realizan en los departamentos de Quindío, Risaralda y Caldas. Algunos investigadores han realizado estudios sobre el sector artesanal desde diferentes campos como el diseño, la administración o la economía. En este contexto, la presente investigación es relevante en cuanto al registro de oficios y técnicas artesanales, los cuales en muchos casos, se están perdiendo, siendo importante resaltarlos haciendo un registro de los mismos y una divulgación a la sociedad, para posibilitar la valoración de este patrimonio.

Se tienen como fundamento, investigaciones previas del Grupo de Investigación de la Facultad de Arquitectura y Diseño de la Universidad Católica de Pereira, Colombia, en cuanto a Bien Mueble Cafetero (García, 2008) y Cultura Material Cafetera (Gómez, 2010 y 2014) y ahora se busca contribuir al conocimiento de los Oficios del Paisaje Cultural Cafetero.

La actual investigación se ocupa de una porción de dicha cultura en lo que se refiere a los oficios, en algunos casos destinados a la producción de objetos y en otros a la construcción, o a la realización de actividades humanas y económicas basadas en las técnicas transmitidas generacionalmente o aprendidas a través de la enseñanza impartida por instituciones.

El proyecto tiene su justificación a partir de la emisión de la Política de Fortalecimiento de los Oficios por parte del Ministerio de Cultura de Colombia en el año 2018, la cual tiene como objetivo general, abrir espacios y oportunidades de valoración y reconocimiento político, social y económico, que favorezcan a los oficios del sector de la cultura desde su desarrollo social, productivo, administrativo, de gestión y de sostenibilidad.

A partir de la expedición de la ley 1834 de la Economía Naranja en 2017, se busca promover las industrias culturales y creativas, de la cual hacen parte las artesanías como un sector que no solamente aporta a la identidad, cohesión social y al fortalecimiento del tejido social y del patrimonio cultural del país; sino que es un potencial generador de crecimiento económico (Mincultura, 2015).

A la vez, el Paisaje Cultural Cafetero Colombiano llega a hacer parte de la lista de Patrimonio de la Unesco mediante la decisión 35 COM8B.43 (FNC-Ministerio de Cultura de Colombia, 2016, p.5) porque cuenta con un Valor Universal Excepcional que gira alrededor de la producción de café, en una región que ha tejido importantes redes de trabajo que garantizan a las futuras generaciones la preservación de una identidad propia, que valora y difunde el patrimonio cultural, apoya la conservación de los recursos naturales y fortalece a través del gremio cafetero, el capital social estratégico. (FNC-Ministerio de Cultura de Colombia, 2016, p.1). 
El Ministerio de Cultura de Colombia, reconoció a través de la Resolución 2079 del 7 de octubre de 2011, el Paisaje Cultural Cafetero como Patrimonio Cultural de la Nación y como un bien inscrito en la Lista de patrimonio mundial de la Unesco, definido como un territorio compuesto por zonas de especial interés arqueológico, histórico y cultural. (FNC-Ministerio de Cultura de Colombia, 2016, p. 5).

Se destacan cuatro valores excepcionales del PCCC, 1.) Esfuerzo humano, familiar, que ha pasado de generación en generación, para producir un café de excelente calidad, 2.) Cultura cafetera para el mundo conformada por diferentes manifestaciones materiales e inmateriales, las cuales involucran elementos arquitectónicos, tradiciones, fiestas, objetos de uso cotidiano, que por años han caracterizado a las poblaciones de esta región. 3.) Capital social estratégico, pues desde 1927 la Federación Nacional de Cafeteros es la entidad encargada de manejar la caficultura del país, a través de sus comités regionales donde los productores participan activamente en la toma de decisiones y 4.) Conservación y equilibrio entre tradición y tecnología para garantizar la calidad y sostenibilidad del producto.

Este territorio se desarrolla principalmente en las laderas de las cordilleras Central y Occidental las cuales pertenecen a la gran cordillera de los Andes. Lo conforman 141.120 hectáreas en zona principal de las cuales 140.046 son del área rural y 1.074 del área urbana.

Forman parte del PCCC, 858 veredas de 51 municipios, 47 en el área principal, 4 en la zona de amortiguamiento y 4 departamentos (Caldas, Quindío, Risaralda y Valle del Cauca) con 24.000 fincas en las que viven de la caficultura 80.000 personas.

Caldas cuenta con 17 municipios en el PCCC: Aguadas, Anserma, Aranzazu, Belalcázar, Chinchiná, Filadelfia, La Merced, Manizales, Neira, Pácora, Palestina, Riosucio, Risaralda, Salamina, San José, Supía y Villamaría con 51.278 ha de área principal, con 159 veredas. En la zona de amortiguamiento se encuentran veredas del municipio de Viterbo. (FNC-Ministerio de Cultura de Colombia, 2016).

Quindío en el PCCC tiene 11 municipios con un total de 27.476 hectáreas en el área principal. 70 veredas. 38.568 hectáreas en el área de amortiguamiento con 58 veredas. Armenia, Buenavista, Calarcá, Circasia, Córdoba, Filandia, Génova, Montenegro, Pijao, Quimbaya y Salento.

Risaralda cuenta con 10 municipios en el PCCC con 32.537 hectáreas de área principal, 108 veredas. Apia, Balboa, Belén de Umbría, Guática, La Celia, Marsella, Pereira, Quinchía, Santa Rosa de Cabal, Santuario y 2 municipios, Dosquebradas y Mistrató de 49.536 hectáteras de área de amortiguamiento, 133 veredas.

El Valle del Cauca cuenta en el PCCC con 10 municipios; 9 municipios con 29.828 hectáreas de área principal con 74 veredas de Alcalá, Ansermanuevo, Caicedonia, El Águila, El Cairo, Riofrío, Sevilla, Trujillo y Ulloa y 1 municipio Argelia en su área de amortiguamiento.

El Valle del Cauca tiene 47.369 hectáreas de área de amortiguamiento con 91 veredas.

\section{Metodología}

El componente principal de la investigación es el método cualitativo. El estudio presenta una visión de los fenómenos e impactos de los oficios por el paso de los años. De esta manera se medirán y evaluarán aspectos cualitativos de la experiencia en los participantes a través de la recopilación de sus historias de vida.

El presente artículo recoge el desarrollo de la primera etapa del proyecto de investigación Oficios del Paisaje Cultural Cafetero Colombiano, el cual adelantan los equipos de investigadores de la Universidad Católica de Pereira, la Universidad de Caldas y la Universidad La Gran Colombia, sede Armenia, en alianza con la entidad 
gubernamental Artesanías de Colombia. En esta etapa se recogen los antecedentes de los estudios relacionados con los oficios del PCCC para determinar sus hallazgos, acudiendo a la información de fuentes secundarias desarrolladas por los Laboratorios de Diseño de Artesanías de Colombia de Caldas, Quindío y Risaralda, publicaciones en la temática, revisión de trabajos de grado y charlas con expertos para establecer un estado del arte de los oficios en este territorio.

El diseño del proceso de investigación cualitativa se basa en la teoría fundamentada (Creswell, 2013), cuyo propósito es desarrollar teoría soportada en datos empíricos y entender los procesos sociales que tienen lugar en ambientes naturales. En la segunda etapa de la investigación se verifican fuentes primarias mediante el trabajo de campo en los municipios seleccionados de Caldas, Quindío y Risaralda, que pertenecen al Paisaje Cultural Cafetero Colombiano; con el fin de documentar historias de vida, realizar observación y recopilación de información a través de entrevistas, registradas mediante videos y fotografías.

En ese caso, la muestra se realiza con los casos disponibles a los cuales tienen acceso los investigadores, además de representar maestros expertos que por su idoneidad, experiencia y desempeño en el oficio se buscan para tal fin, así mismo de lo referenciado por funcionarios de Artesanías de Colombia que han trabajado anteriormente con ellos. Las personas en su gran mayoría son adultas.

\subsection{Procedimiento}

Durante la primera etapa se pretende entender los detalles, los actores e información significativa que se pueda encontrar en estudios previos en cuanto a historias de vida de los hacedores de los oficios, el desarrollo de los procesos, las técnicas y los materiales utilizados, así como los productos elaborados en los municipios estudiados.

\subsection{Recolección y análisis de datos}

Se busca obtener datos que se convertirán en información y conocimiento. Los autores cuentan con un instrumento a manera de ficha bibliográfica para recopilar la información de historias de vida y de los oficios; siendo las herramientas más importantes los documentos bibliográficos, artículos, trabajos de grado, videos, exposiciones fotográficas, registros y artefactos.

Los análisis se inician con la estructuración de datos, a través de la organización de los mismos, mediante la clasificación y selección del material, lo anterior se consigna en un informe a manera de bitácora de análisis, para documentar el proceso, que dará lugar a interpretar datos para la generación de explicaciones y teorías.

\subsection{Aplicación de los instrumentos}

A partir de la revisión documental se reconocen las características de los grupos humanos dedicados a los oficios, las condiciones y la manera en que los desarrollan para identificar patrones o vinculaciones alrededor de los oficios artesanales.

\subsection{Charlas con expertos}

Como parte de la recopilación de información se realiza una serie de sesiones expositivas con los funcionarios de los Laboratorios de Diseño de Artesanías de Colombia con el fin de reconocer desde su experiencia a las personas que se dedican a determinados oficios en el PCCC y los desarrollos que en su interacción han alcanzado con ellos.

\section{Resultados - Disusión de resultados}

En el caso del departamento de Risaralda se tuvo acceso a varios documentos del Repositorio de la Biblioteca Digital de Artesanías de Colombia, los cuales están organizados por temáticas: geográfica, por oficio, por técnicas o por productos. 
Dos estudios con información significativa realizados por Artesanías de Colombia en los años 1999 "Diagnóstico de Desarrollo Artesanal Departamento de Risaralda Municipios de Apia, Quinchía, Balboa, Santa Rosa De Cabal y Zona Indígena (Mistrató y Pueblo Rico - Santa Cecilia)" y 2015 "Fortalecimiento integral del sector artesano del departamento de Risaralda: Informe final", permiten dar cuenta de lo registrado a nivel de oficios en este departamento.

A través de estos informes se pudo establecer que en el municipio de Apía se encuentra que en 1999 hay una mención importante a la cestería en caña brava y calceta de plátano, no así en el 2015 donde se hace mención a los trabajos en madera y sobre todo los productos de mobiliario. Lo que parece coincidir con otras investigaciones donde la cestería se ha reducido en varios municipios de Risaralda.

En Quinchía, en el trancurso de esos 16 años se pasó de contar con artesanos de joyería con potencial considerable,con mano de obra regular, materias primas fijas y un bajo grado de tradición, a unidades productivas dedicadas al oficio de la joyería en filigrana, beneficiaria de proyectos en los que se han implementado conceptos de diseño y desarrollo de producto, calidad, comercialización, generando así mismo, reconocimiento y vigencia.

En Santa Rosa de Cabal, se registra en 1999 que los artesanos trabajan la madera produciendo elementos de menaje para la cocina, talla, calados y la mayoría realizaban todos los mismos diseños, sin una línea de estilo propio. Con el paso de los años hacia el 2015, los artesanos han sido influenciados por diseñadores del Laboratorio de Diseño de Risaralda, que han aportado al sector una diversificación importante en los productos, así como mejoramiento en los procesos y la calidad. Hay una evolución significativa en el manejo de color, acabados y en especialización de productos.

En el informe de 1999, se presenta el caso de la artesanía indígena Embera en Mistrató y Pueblo Rico (Municipios que no pertecenen al PCCC), donde en el oficio de la cestería hay especialización por sexos, algunos cestos son elaborados exclusivamente por los hombres y otros por las mujeres, aunque es una actividad en donde predomina el género femenino. Son utilizadas las raíces aéreas de algunas plantas como el sinú, la corteza de iraca, la conga, el joro, la palma amarga, la guadua y la caña brava. Se registra que los detalles y acabados de los productos de cestería son de baja calidad y carecen de técnicas en tinturación. En el informe del 2015 no se hace relación al caso de la artesanía Embera.

En el informe de 2015, se relata lo conceriente a cinco municipios más; Belén de Umbría, con una comunidad dedicada a la producción de la materia prima de la seda, mediante cultivos en zonas rurales hasta la transformación y comercialización. La asociación Arte y Seda ha sido beneficiaria de proyectos y capacitación en conceptos de diseño de producto, calidad y técnicas como crochet, malla, punto de cruz y macramé, pero requiere implementar procesos creativos y fundamentos de estética.

Dosquebradas; cuenta con una de las comunidades artesanales más grandes del departamento, con oficios tradicionales como trabajos en madera, guadua y un sector dedicado a las artes manuales. No tienen dificultades para agruparse, atienden las convocatorias de las entidades desarrolladoras de proyectos y encuentran en la Alcaldía y Cámara de Comercio que les proporcionan herramientas para el desarrollo del sector. Tienen falencias en cuanto a su intermitencia en diferentes oficios, razón por la cual pierden enfoque y direccionamiento.

En Guática, la comunidad artesanal se caracteriza principalmente por el oficio de la seda, desde el cultivo, la transformación de la materia prima y comercialización de sus productos textiles. El gremio es bastante participativo pues cuenta con líderes que se han hecho visibles por su perseverancia y trabajo duro en el oficio. Lo anterior produce que hoy en día se tenga como referente el oficio de la seda en el departamento. 
La Celia; la población artesana del municipio está dividida entre manualidades como trabajos en calceta de plátano y penca de fique, entre otros. Igualmente se encuentra un caso exitoso dedicado al oficio de trabajos en madera (mobiliario rústico).

Santuario, la mayoría de los artesanos en el municipio trabajan manualidades con insumos de reciclaje por tanto, las asesorías se han orientado hacia la calidad y estética de los productos para promover una producción más cuidadosa.

\subsection{Otras investigaciones de los oficios en Risaralda}

Se han encontrado otros estudios de oficios en el departamento de Risaralda que se sintetizan en la tabla 1, a partir de lo cual, se puede establecer que se mantienen vigentes hasta el día de hoy algunos oficios como la cestería, referenciada en dos investigaciones y la joyería en filigrana, en territorios como Santa Rosa de Cabal y Quinchía respectivamente. Son investigaciones puntuales enfocadas a un solo oficio y en un determinado lugar.

Tabla 1

Otras investigaciones de los oficios en Risaralda

\begin{tabular}{|c|c|c|}
\hline $\begin{array}{c}\text { Autores: Linares, Galeano, García y } \\
\text { Figueroa }\end{array}$ & $\begin{array}{c}\text { Año: 2008, Oficio: Cestería y } \\
\text { sombreduría }\end{array}$ & Municipio: Varios \\
\hline
\end{tabular}

La investigación documenta a lo largo del estudio de las fibras naturales utilizadas en la artesanía, los oficios relacionados con el aprovechamiento de estas fibras en el Eje Cafetero como la cestería y la sombreduría y los productos elaborados con diferentes especies.

\begin{tabular}{l|l|l}
\hline Autor: Pérez & Año: 2010, Oficio: Talla & Municipio: Santa Rosa
\end{tabular}

Hace un análisis de las formas de los objetos elaborados en talla de madera alrededor de lo religioso, lo costumbrista, lo utilitario y lo artístico. En su análisis concluye que: El oficio del tallador surge del proceso de aprendizaje dado por la interacción con actividades relacionadas con la madera y, en particular, por las enseñanzas asumidas a través de sus antecesores, con lo cual se puede evidenciar que, es ante todo un hacer que nace paulatinamente con la experiencia en el manejo de la madera. (P. 82)

\begin{tabular}{l|l|l} 
Autores: Tinjacá, Slone y Arango & Año: 2012, Oficio: Fique & Municipio: Pueblo Rico
\end{tabular}

El estudio toma como caso la Asociación de Artesanos Fiqueros y Asociación de Artesanos de la Cabuya ASDEFIARCA de Pueblo Rico que se dedica a la producción y comercializa- ción de la fibra y la elaboración de productos artesanales en fique como bolsos, accesorios, calzado y productos para el hogar. Se documenta el proceso productivo del fique y los productos que se elaboran, analizando el mercado y encontrando oportunidades de diseño para el sector moda definiendo algunas propuestas de productos para la asociación. Es de aclarar que el libro documenta este oficio en Risaralda, pero el municipio de Pueblo Rico no hace parte del PCCC.

\begin{tabular}{|c|c|c|}
\hline Autor: Suárez & Año: 2013, Oficio: Filigrana & Municipio: Quinchía
\end{tabular}

La autora establece que: En los últimos 20 años se han consolidado dos asociaciones de joyeros, apoyadas por diferentes programas y estrategias hacia el fortalecimiento de la actividad productiva. En Pereira una actividad joyera importante, caracterizada por pequeñas unidades apoyadas con programas de formación de técnicos joyeros, sin embargo, el diseño como mecanismo de generación de valores agregados no se incluye como parte integral en la formación de los aprendices. Generalmente, los modelos fabricados son copias o reproducciones de catálogos internacionales limitando la capacidad competitiva de Risaralda. (p. 48-49).

\begin{tabular}{|l|c|c|}
\hline Autor: Arias & Año: 2016,Oficio: Cestería & Municipio: Santa Rosa \\
\hline Registra que en el Eje Cafetero, la cestería data hace un poco más de 150 años, como un oficio forjado por las familias \\
asentadas durante la colonización antioqueña en la elaboración de cestos útiles para actividades cotidianas. (P. 28). La \\
cestería en la cultura campesina, tuvo su auge en la época de la bonanza cafetera de la primera mitad del siglo XX. Hasta \\
hace 40 años, era común encontrar artesanos tejiendo y exponiendo sus canastos de bejuco en las plazas de los pueblos \\
del Eje Cafetero. La crisis de la comercialización llevó a la deserción de la mayoría de familias y artesanos cesteros de este \\
oficio. Los cestos tradicionales actualmente son apreciados como un símbolo de la cultura cafetera de antaño. (p. 30). \\
Aparte de Santa Rosa, hoy se evidencia en Risaralda, la cestería como una actividad en vía de extinción.
\end{tabular}

Fuente: Elaboración Propia 
La búsqueda para establecer un balance bibliográfico en la Biblioteca del Banco de la República sede Pereira, permitió identificar 41 publicaciones sobre la temática de PCCC y algunas referidas a oficios.

Frente a la descripción de los procesos de los oficios se encontró en la Caracterización Ocupacional del Subsector de Artesanías de la Mesa de Artesanías (2012), el registro descriptivo del paso a paso del oficio de tejeduríacestería, de la elaboración de objetos artesanales en madera y sus acabados, del trabajo en guadua, de las artesanías en cuero y de las artesanías en joyería; no obstante no necesariamente delimitados al contexto del PCCC, sino en general a su presencia en el país y los modos de hacer.

\subsection{Historias de vida}

Las historias de vida de algunos artesanos de los municipios del Paisaje Cultural Cafetero Colombiano, están referenciadas en las publicaciones de Artífices de Artesanías de Colombia así como en el libro de Maestros del Arte Popular (2014) y en el de Oficios de Manos Colombianas (2017), en ellos se cuenta con la recopilación de varios exponentes de oficios a nivel del país recogiendo algunos presentes en el PCCC, como el caso del trabajo de la guadua.

De igual manera se encontraron algunos videos realizados por la Gobernación de Risaralda o por el Ministerio de Cultura donde se documentan algunas historias de vida de artesanos.

En la búsqueda de antecedentes de los oficios del Paisaje Cultural Cafetero Colombiano, se encuentran valiosos exponentes de diferentes técnicas que enriquecen la cultura material en la zona cafetera en mención. Se relacionan artesanos de los cuales se ha publicado su obra y otros identificados según las fuentes de información proporcionadas por los Laboratorios de diseño de Caldas, Quindío y Risaralda.

En el caso del departamento de Risaralda se encontraron algunas reseñas de las historias de vida que se recogen en la tabla 2. De lo cual se evidencia que la institución de Artesanías de Colombia ha procurado hacer visible la experiencia personal de los artesanos, a través de narraciones breves que exponen las particularidades de cada personaje y su labor.

Tabla 2

Historias de vida reseñadas

\begin{tabular}{|c|c|}
\hline Artesana Gloria Bayer & \\
\hline \multicolumn{2}{|c|}{$\begin{array}{l}\text { Maestra artesana de la seda, recibió capacitación en el oficio y se ha caracterizado por la calidad y estética de sus } \\
\text { productos. }\end{array}$} \\
\hline Artesano Edilson Tanigama & \\
\hline \multicolumn{2}{|c|}{$\begin{array}{l}\text { Es un Artesano Indígena que ha realizado un importante trabajo haciendo visible la etnia Embera Chamí a través de su } \\
\text { liderazgo en la asociación Jaipono y su buen desempeño en las redes sociales, que lo han hecho un referente } \\
\text { internacional. }\end{array}$} \\
\hline Artesano Luis Octavio Ocampo & \\
\hline \multicolumn{2}{|c|}{ Rosa, exponente del oficio de trabajos en madera,ha proyectado su empresa a } \\
\hline Artesano Uber Lemus & \\
\hline \multicolumn{2}{|r|}{$\begin{array}{l}\text { muebles. Las personas conocen la técnica, pero no la identifican con el } \\
\text { os productos elaborados con la técnica de la cestería por la falta de } \\
\text { erdiendo y olvidando por la difícil adquisición de la materia prima ya } \\
\text { jetivo la conservación del bosque. }\end{array}$} \\
\hline
\end{tabular}

Fuente: Elaboración Propia 


\subsection{Video}

En la búsqueda de información en videos relacionados, se encontraton algunos referidos a los oficios, Tabla 3, y otros al entorno del Paisaje Cultural Cafetero Colombiano Tabla 4. Donde se puede observar que hay un mayor empoderamiento de los entes gubernamentales locales como la Gobernación y Artesanías de Risaralda para registrar la memoria histórica visual de los oficios, por medio la realización de los videos alusivos a los artesanos que han apoyado. Mientras para el registro del tema de Paisaje Cultural Cafetero, es el Ministerio de Cultura, la institución que documenta en los videos el valor de este patrimonio, aunque no se incluyen de manera significativa las artesanías.

Tabla 3

Videos acerca de oficios en el PCCC

\begin{tabular}{|c|c|c|}
\hline Autor: Artesanías de Colombia y Gobernación de R/lda & Año: 2016 & Duración: 6:17 min \\
\hline \multicolumn{3}{|c|}{$\begin{array}{l}\text { Contenido: En el video hablan William Uribe Vélez y Carlos Uribe de la empresa Arte Decorativo Pinares de Santa Rosa de } \\
\text { Cabal. }\end{array}$} \\
\hline Autor: Ministerio de Cultura & Año: 2017 & Duración: 5:10 min \\
\hline \multicolumn{3}{|c|}{$\begin{array}{l}\text { Contenido: Colección Memoria Viva Oficios con } 27 \text { historias audiovisuales, sobre el patrimonio cultural de Colombia que } \\
\text { dan cuenta de la importancia de la transmisión de este conocimiento. Aunque ninguno de estos videos documenta algún } \\
\text { oficio del PCCC. }\end{array}$} \\
\hline Autor: Artesanías de Risaralda & Año: 2018 & Duración: 46 seg \\
\hline \multicolumn{3}{|c|}{ Contenido: Video de Gloria Bayer de Guática, acerca de la elaboración de productos en seda } \\
\hline Autores: Artesanías de Risaralda y Gobernación de R/lda & Año: 2019 & Duración: 1:20 min \\
\hline \multicolumn{3}{|c|}{ Contenido: Se narra cómo los finos hilos de diversos metales se transforman en piezas de altísima maestría joyera. } \\
\hline Autores: La Lucerna y Artesanías de Colombia & Año: 2020 & Duración: 5:44 min \\
\hline \multicolumn{3}{|c|}{$\begin{array}{l}\text { Contenido: Desarrollo de una serie de empaques donde se registra a varios artesanos de Caldas, Valle del Cauca y Quindío, } \\
\text { quienes se dedican a trabajos en tejido de Iraca, oficios en madera y chaquiras, cestería en guadua y en caña brava, trabajo } \\
\text { en cáscara de huevo y arcilla. }\end{array}$} \\
\hline
\end{tabular}

$$
\text { Fuente: Elaboración Propia }
$$

Tabla 4

Otros videos acerca del Paisaje Cultural Cafetero

\begin{tabular}{|c|c|c|}
\hline Autores: U. de Antioquia, Ministerio de Cultura & Año: 2012 & Duración: 52 min \\
\hline \multicolumn{3}{|c|}{$\begin{array}{l}\text { Título: Soy Patrimonio Paisaje Cultural Cafetero. Capítulo } 1 \text { de la serie "Expreso Colombia. Un país que vive su cultura". } \\
\text { Entrevistas a expertos que relatan acerca de la declaratoria de patrimonio cultural de este territorio, su significado y } \\
\text { valoración, la experiencia de la vida cafetera, el proceso cultivo y transformación del café. Otros expertos hablan acerca de } \\
\text { las costumbres, los mitos y las leyendas, así como los cuentos de arrieros. }\end{array}$} \\
\hline Autores: U. Antioquia, Ministerio de Cultura & Año: 2012 & Duración: 52 min \\
\hline \multicolumn{3}{|c|}{$\begin{array}{l}\text { Título: Soy Patrimonio Paisaje Cultural Cafetero. Capítulo 2. serie "Expreso Colombia". Con el título de "Así se vive el Paisaje } \\
\text { Cultural Cafetero", se presenta el transporte en jeep y varios objetos de anticuario que hicieron parte de la vida doméstica } \\
\text { y del campo. }\end{array}$} \\
\hline Autor: Ministerio de Cultura & Año: 2017. & Duración: 5:40 min \\
\hline
\end{tabular}

Fuente: Elaboración Propia

\subsection{Sesión expositiva con expertos}

Se realizó por parte del equipo investigador, un encuentro con los diseñadores industriales del Laboratorio de Diseño de Artesanías de Colombia en Risaralda, Alejandro Jaramillo Suárez y Daniel Amariles Zapata. Para 
sustentar su exposición, muestran la bitácora de diversos municipios en donde se tiene información de artesanos y talleres artesanales:

En el municipio de Santa Rosa se realizan múltiples oficios como trabajos en madera, tejeduría en telar, cestería. Los diseñadores exponen el documento y refieren que aunque la vocación artesanal de este municipio es significativa, los hacedores de oficios no se agrupan fácilmente. En el área de trabajos en madera se cuenta con el espacio de "quioscos" del Barrio de Los Artesanos, donde se encuentran talleres y maquinaria para trabajar, sin embargo, raramente se unen para trabajar allí, pues los artesanos prefieren ubicarse en sus talleres personales y familiares.

Los diseñadores también relacionan algunos artesanos representativos del municipio: ASEDAR en el barrio EI Vergel; Carlos Alberto López, artesano consagrado al trabajo en madera; Alejandro Mesa quien dedica al mobiliario de cocina y productos en guadua y ACESTERISA, Asociación de Artesanos de la Cestería de Santa Rosa, en donde Fredy Morales es un miembro activo e interesado en impulsar el oficio (Artesanías de Colombia, 2018).

En el ámbito de la cestería, se manifiestan dificultades que experimentan los artesanos al conseguir la materia prima ya que alrededor del bejuco hay muchas restricciones y preocupaciones en general. Una gran cantidad de personas que se encargan de obtener la materia prima son de edades muy avanzadas y no utilizan elementos de protección mínimos para esta labor. Los diseñadores también hace mención de la apropiación de métodos de tinturación aplicados a las fibras naturales por parte de los artesanos tejedores en el eje cafetero, siendo más experimentados en el Quindío.

En el municipio de Guática, la convocatoria artesanal es de poca asistencia, pero los que se reúnen, hacen un proceso colaborativo con las entidades capacitadoras. Se encuentra la asociación Café y Seda quienes realizan el oficio con el más mínimo de los cuidados de la seda de principio a fin. Tejen en telar liderados por la maestra artesana Gloria Bayer, reconocida por su destreza en el oficio y la variedad de productos exitosos. Una de las debilidades en esta comunidad es la consecución de materia prima pues cuando no tienen suficiente acceso a ella, la buscan en comunidades como en el Cauca.

Se mencionan las comunidades Embera Chamí radicadas en varios municipios, quienes desarrollan tejeduría en chaquira mostacilla checa ${ }^{3}$. Los indígenas realizan diversos productos como collares, pulseras, aretes y Okamas (collares de alto contenido simbólico) que muestran su cosmovisión a la gente. Los Laboratorios de Artesanías de Colombia han trabajado en el "rescate" tanto de sus tradiciones como de su cosmovisión y su manera de elaborar el producto, sin perder sus valores ancestrales. Nunca se imponen nuevos productos, siempre se rescatan sus objetos principales asociados a sus creencias y forma de vida.

De otra parte, según el Informe para el Taller de Construyendo País llevado a cabo en Pereira en abril de 2019, Artesanías de Colombia señala que: "Los principales oficios artesanales del departamento de Risaralda son: bisutería indígena de la comunidad Embera Chamí, y de artesanos tradicionales productos de joyería, tejeduría, cerámica, bisutería, trabajo en madera, telar horizontal, marroquinería, seda, cestería, fique y calceta de plátano. Se requiere fortalecer la actividad artesanal aprovechando la vocación turística del departamento".

3 La mostacilla checa es un producto para la elaboración de bisutería, en especial de pulseras y collares, deriva su nombre de Europa de la república Checa,es elaborada a partir de cristales de vidrio fundidos a altas temperaturas y vertidos en moldes de acero, con adición de pigmentos. Es fabricada por la empresa Preciosa Hornella. Esta mostacilla es muy uniforme y diferente de la chaquira china la cual es plástica y es de menor calidad. 


\subsection{Características sociodemográficas de la población artesanal de risaralda}

Entre 2014 y 2018, el Sistema de Información Estadístico de la Actividad Artesanal (SIEAA) ha logrado identificar y caracterizar 1.343 artesanos en el departamento de Risaralda, en 10 municipios de los 14 del departamento, donde el $67 \%$ son mujeres y el $33 \%$ hombres ubicados en su mayoría $68,4 \%$ en zona urbana, un $12,1 \%$ en zona rural y un 19,5\% en resguardo indígena. El 39\% de dicha población corresponde a población indígena y un $1 \%$ afrodescendiente y un $60 \%$ no corresponde a ningún grupo étnico. En su gran mayoría son trabajadores independientes que realizan sus ventas de manera directa, donde solo un $22 \%$ de ellos participa en ferias artesanales.

El $42 \%$ de los núcleos familiares están compuestos por 3 o 4 personas, el $56 \%$ de los hogares de los artesanos reciben mensualmente, en promedio, menos de un Salario Mínimo Mensual Legal Vigente. El $47 \%$ de los artesanos se considera en condición de vulnerabilidad.

\section{Conclusiones}

Se llega a la información de varios de los oficios presentes en el Paisaje Cultural Cafetero Colombiano a través de la literatura de las materias primas, como es el caso de las fibras naturales, de investigadores de las ciencias naturales y de la administración del medio ambiente. También se llega a los oficios a través de los registros encontrados de historias de vida de algunos artesanos, de los testimonios de expertos que han trabajado con ellos en esta zona y de libros referidos a la artesanía en Colombia. A través del video, ha habido un interés de parte de las instituciones gubernamentales locales para registrar los oficios.

La información encontrada en la Biblioteca Digital de Artesanías de Colombia en su mayoría, corresponde a informes de los Laboratorios de Diseño e Innovación, hallando contenidos de manera fragmentada en documentos que no han sido elaborados con un rigor científico, que permitan determinar datos suficientes para dar cuenta integralmente de los oficios y su forma de ejecución en el PCCC. No obstante, se encuentra una relevante cantidad de información frente a la cestería y el trabajo en guadua. Las historias de vida documentadas fueron escasas, a la vez se observó que son pocos los casos en los que se realizan los oficios conservando la tradición artesanal.

Lo documentado hasta el momento permite evidenciar que instituciones como Artesanías de Colombia y sus Laboratorios de Diseño e Innovación, el Servicio Nacional de Aprendizaje SENA, la Gobernación de Risaralda, Artesanías de Risaralda y la Corporación Autónoma Regional de Risaralda CARDER, han sido agentes claves para el desarrollo y reconocimiento de los oficios en el departamento, a través de sus diferentes programas de capacitación y fortalecimiento al sector artesanal.

Frente a la caracterización de la población artesanal en Risaralda, se evidencia el mayor porcentaje de participación de la mujer en la realización de oficios, la manifestación de la vulnerabilidad de esta población, siendo un 19 \% indígena. La comunidad artesanal en el departamento ha presentado una evolución significativa durante los últimos años, en la configuración de sus emprendimientos desde la planeación de la producción hasta la presentación en espacios comerciales. Lo anterior se ha dado como resultado a la representación y búsqueda de oportunidades para el sector artesanal por parte de los entes encargados y la activa participación de los artesanos al dejarse instruir, capacitar y guiar a través de los beneficios del sector.

Risaralda se ha posicionado a nivel nacional gracias a oficios como el proceso y tejeduría en seda del municipio Guática y la tejeduría en mostacilla checa de comunidades artesanales Embera Chamí, así como el trabajo de la madera en Santa Rosa de Cabal. El alto nivel de destreza para desarrollar las diversas técnicas de los oficios, ha sido un referente para el reconocimiento de estas comunidades. No obstante, se evidencia que cada vez es menor el relevo generacional y que varios oficios están en riesgo de desaparecer. 
En los municipios de Santa Rosa y Dosquebradas, se puede apreciar cómo los oficios tradicionales han sido llevados a un nivel superior gracias a la práctica y perseverancia de algunas unidades productivas que se han mantenido vigentes durante los últimos 15 años.

La industria de la moda ha impactado significativamente el sector artesanal en Risaralda gracias a la apertura que tienen los artesanos para aplicar las tendencias en diseño, color, nuevos métodos de producción y adaptarse al mundo de las redes sociales para promocionar este tipo de productos.

\section{Referencias bibliográficas}

Arias, D. (2016). Lineamientos para el aprovechamiento sostenible de plantas silvestres productoras de fibras vegetales útiles para cestería en el departamento de Risaralda. Recuperado de http://repositorio.utp.edu.co/dspace/bitstream/handle/11059/6845/33372A696.pdf?sequence=1\&isAllo wed=y

Artesanías de Colombia. (1999). “Diagnóstico de Desarrollo Artesanal Departamento de Risaralda Municipios de Apia, Quinchía, Balboa, Santa Rosa De Cabal Y Zona Indígena (Mistrató y Pueblo Rico - Santa Cecilia)" Recuperado de https://repositorio.artesaniasdecolombia.com.co/bitstream/001/2397/1/INSTD\%201999.\%2012.pdf

Artesanías de Colombia. (2014). Maestros del arte Popular Colombiano. Bogotá.

Artesanías de Colombia. (2015). Fortalecimiento integral del Sector Artesano del Departamento de Risaralda 2015 Informe Final. Pereira: Artesanías de Colombia.

Artesanías de Colombia. (sf). El sector artesano en Colombia. Bogotá, Colombia: Artesanías de Colombia S.A. Recuperado de http://artesaniasdecolombia.com.co/PortalAC/C_sector/caracterizacion_81

Artesanías de Colombia. (2017, semestral). 4. Tejiendo Redes. Artífices No 9. Recuperado de https://repositorio.artesaniasdecolombia.com.co/handle/001/4047

Artesanías de Colombia. (2017, semestral). 18. Ese Gusano de Seda. Artífices No 7. Recuperado de http://repositorio.artesaniasdecolombia.com.co/handle/001/3774

Artesanías de Colombia. (s.f) Características sociodemográficas de la población artesanal de Risaralda. Recuperado de http://www.artesaniasdecolombia.com.co/Documentos/Contenido/32010_risaralda.pdf

Artesanías de Colombia (2018) Diagnóstico de oficios Laboratorio de Diseño e Innovación Risaralda 2018 Recuperado de https://repositorio.artesaniasdecolombia.com.co/bitstream/001/4652/3/INSTD\%202018.\%2085.\%202.pdf

Artesanías de Colombia. (2018, semestral). 8. El Juego de Madera. Artífices No 11. Recuperado de https://repositorio.artesaniasdecolombia.com.co/handle/001/4168

Bejarano, E. (2014). Artesanías y Sistemas de Información. En: Investigaciones y productos CID N²2. Bogotá: Ed. Centro de Investigaciones para el Desarrollo. Universidad Nacional de Colombia.

Congreso de la República de Colombia. (2017). Ley 1834. La economía creativa ley naranja. https://dapre.presidencia.gov.co/normativa/normativa/LEY\%201834\%20DEL\%2023\%20DE\%20MAYO\%20 DE\%202017.pdf 
Creswell, J. W. (2013). Qualitative Inquiry \& Research Design Choosing among Five Approaches. (3rd ed.). Thousand Oaks, CA SAGE.

Duque, C., y Artesanías de Colombia. (2014). Maestros del arte popular. Bogotá: Ed. Panamericana

García, L. (2008). Bien Mueble Cafetero. En: Paisaje Cultural Cafetero. Risaralda Colombia. Pereira: Ed: Universidad Católica Popular del Risaralda. Universidad Tecnológica de Pereira, CARDER, Gobernación de Risaralda, Ministerio de Cultura, Federación Nacional de Cafeteros. (PP.171-205).

Gómez, Y. (2010). El Estado del Arte la Cultura de la Cultura Material Cafetera. Revista Arquetipo, No. 2 Pereira: Ed. Universidad Católica de Pereira (PP.39-53).

Gómez, Y. (2013). Cultura Material Cafetera. Colección Maestros No. 16. Pereira: Ed.Universidad Católica de Pereira.

Jaramillo, L. (2018). Diseño de línea de bolsos y calzado a través del uso de la cestería tradicional. Universidad Católica de Pereira. Recuperado de http://repositorio.ucp.edu.co/bitstream/10785/5162/2/DDMDI53.pdf

Linares, E., Galeano, G., García N., y Figueroa, G. (2008). Fibras vegetales utilizadas en artesanías en Colombia. Bogotá: Ed. Artesanías de Colombia, Ministerio de Comercio, Industria y Turismo, Instituto de Ciencias Naturales, Facultad de Ciencias - Universidad Nacional de Colombia, Sede Bogotá.

Ministerio de Cultura. (2018). Política para el fortalecimiento de los oficios culturales. Bogotá.

Ministerio de Cultura. (2015). Cultura a la medida. Análisis de la Cuenta Satélite de Cultura de Colombia. Recuperado de http://sicsur.mercosurcultural.org/archivos/Cultura-a-la-Medida.pdf

Ministerio de Cultura y Federación Nacional de Cafeteros. (2016). Paisaje Cultural Cafetero de Colombia. Excepcional fusión entre naturaleza, cultura y trabajo colectivo. Bogotá: UNESCO.

MNR Comunicaciones y ediciones y Artesanías de Colombia. (2017). Oficios de manos colombianas. Bogotá: Panamericana formas e impresos S.A.

Pérez, C. A. (2010). Diseño, Artesanía y Estética. Revista Arquetipo P. 73-82. Pereira: Universidad Católica de Pereira. Recuperado de http://repositorio.ucp.edu.co/bitstream/10785/1208/1/ARQUETIPO\%201.pdf

SENA y Artesanías de Colombia. (2012). Mesa del subsector de Artesanías. Caracterización Ocupacional del Subsector de Artesanías. Sin paginación, recuperado de https://repositorio.artesaniasdecolombia.com.co/handle/001/2471

Suárez, L. (2013). Artesanía: historia, concepto y dinámicas adaptativas a través de la cadena oro-joya. Revista Páginas No. 94: (pp. 39 - 50).

Tinjacá, B., Slone, V., y Arango, N. (2012). El Fique. Fibra natural que construye sociedad. Pereira: Fundación del Área Andina. Recuperado de https://es.slideshare.net/RobelsydMariaGonzale/el-fique

\section{Videos:}

Artesanías de Colombia y Gobernación de Risaralda. (2016). Santa Rosa Municipio Artesanal. Ser artesano es dejar que el alma salga a la luz transformada. Recuperado de: https://www.facebook.com/855022931214033/videos/1100311893351801/

Artesanías de Risaralda y Secretaría de desarrollo económico y competitividad de la Gobernación de Risaralda. (2019). Quinchía, Risaralda. Recuperado de 
https://www.facebook.com/risaraldallenadevida/videos/340017513346428/UzpfSTg1NTAyMjkzMTIxNDAz MzoyMjY4ODM5ODYzMTY1NjU5/

Artesanías de Risaralda (2018). Gloria, B. (Video). Recuperado de

https://www.facebook.com/855022931214033/videos/2271440356417501/

La Lucerna y Artesanías de Colombia. (2020). Alianza de la Lucerna y Artesanías de Colombia.(Video)

Recuperado de https://www.youtube.com/watch?v=tONK_A7c7pg

Ministerio de Cultura. (2017). Memoria Viva. (Video). Recuperado de

https://bancodecontenidos.mincultura.gov.co/AppSeries/RecursosSeries/?idSerie=11729

Quintero Paz, A., y Ramírez Mendoza, L. (2016). La Familia Cafetera. Ministerio de Cultura. Recuperado de https://bancodecontenidos.mincultura.gov.co/FichaDocumental/?id=9735

Universidad de Antioquia y Ministerio de Cultura de Colombia. (2012). Paisaje Cultural Cafetero. Capítulo 1 y 2. Recuperado de https://bancodecontenidos. mincultura.gov.co/FichaDocumental/?id=2432

Esta obra está bajo una Licencia Creative Commons Attribución-NoCommercial 4.0 International

(cc) BY-NC 